\title{
Mortalidade por Covid-19 no Brasil: perfil sociodemográfico das primeiras semanas
}

\author{
Mortality due to Covid-19 in Brazil: sociodemographic profile of the first weeks \\ Mortalidad por Covid-19 en Brasil: perfil sociodemográfico de las primeras semanas
}

Recebido: 29/12/2020 | Revisado: 30/12/2020 | Aceito: 14/01/2021 | Publicado: 17/01/2021

\author{
Elias Ferreira Porto \\ ORCID: https://orcid.org/0000-0001-8326-2054 \\ Centro Universitário Adventista de São Paulo, Brasil \\ E-mail: eliasfporto@gmail.com \\ Alessandro Leipnitz Domingues \\ ORCID: https://orcid.org/0000-0002-8552-2160 \\ Centro Universitário Adventista de São Paulo, Brasil \\ E-mail: domingues.alessandro@bol.com.br \\ Anselmo Cordeiro de Souza \\ ORCID: https://orcid.org/0000-0002-0156-716X \\ Centro Universitário Adventista de São Paulo, Brasil \\ E-mail: anselmo.vivamelhor@hotmail.com \\ Monica Karla Vojta Miranda \\ ORCID: https://orcid.org/0000-0001-9610-0468 \\ Centro Universitário Adventista de São Paulo, Brasil \\ E-mail: monicavojta@hotmail.com \\ Morenilza Bezerra da Conceição Froes \\ ORCID: https://orcid.org/0000-0001-7141-5172 \\ Centro Universitário Adventista de São Paulo, Brasil \\ E-mail: morenilza@hotmail.com \\ Sérgio Rosa Vieira Pasqualinoto \\ ORCID: https://orcid.org/0000-0003-0864-3871 \\ Centro Universitário Adventista de São Paulo, Brasil \\ E-mail: sergioex_3@hotmail.com
}

\begin{abstract}
Resumo
Em 26 de fevereiro de 2020, o Ministério da Saúde confirmou o primeiro caso de óbito por Covid-19 no Brasil. Este estudo objetivou-se identificar a mortalidade pela doença no Brasil nas 6 primeiras semanas após a confirmação do primeiro caso de óbito e traçar o perfil desses indivíduos. Trata-se de um estudo transversal descritivo retrospectivo, que utilizou dados dos boletins diários do Ministério da Saúde. Foram analisados todos os casos de óbitos ocorrido no Brasil por Covid-19, do dia 17 de março até a data de 26 de abril 2020. Foram incluídos dados de todos os estados da federação e caracterizados por sexo, idade, cor da pele e presença de comorbidade associada. Dentre as mortes, $72 \%$ foram de pessoas com mais de 60 anos, embora cerca de $80 \%$ dos infectados não pertencessem a essa faixa etária. Ainda, $60 \%$ eram do sexo masculino, mesmo com média de apenas $51,4 \%$ dos infectados do sexo masculino. A frequência de óbitos foi significantemente maior entre os indivíduos de cor branca em relação aos de cor parda e negra ( $\mathrm{p}<0,0001)$. A taxa de mortalidade foi de $6,92 \%$, sendo maior entre os indivíduos do sexo masculino, os mais idosos principalmente entre aqueles que tinham morbidades associadas - e os de cor branca da pele.
\end{abstract}

Palavras-chave: Covid-19; SARS-CoV-2; Mortalidade; Fatores de risco.

\begin{abstract}
On February 26, 2020, the Ministry of Health confirmed the first case of death by Covid-19 in Brazil. This study aimed to identify mortality from the disease in Brazil in the first 6 weeks after confirmation of the first case of death and to outline the profile of these individuals. This is a retrospective, descriptive cross-sectional study, which used data from the daily bulletins of the Ministry of Health. All cases of deaths occurred in Brazil by Covid-19, from March 17 to April 26, 2020, were analyzed. Data from all states of the federation were included and characterized by sex, age, skin color and presence of associated comorbidity. Among the deaths, $72 \%$ were from people over 60 years old, although about $80 \%$ of those infected did not belong to this age group. Still, $60 \%$ were male, even with an average of only $51.4 \%$ of infected males. The frequency of deaths was significantly higher among white individuals compared to brown and black individuals $(\mathrm{p}<0.0001)$. The mortality rate was $6.92 \%$, being higher among males, the elderly - especially among those who had associated morbidities - and those with white skin.
\end{abstract}

Keywords: Covid-19; SARS-CoV-2; Mortality; Risk factors. 


\begin{abstract}
Resumen
El 26 de febrero de 2020, el Ministerio de Salud confirmó el primer caso de muerte por Covid-19 en Brasil. Este estudio tuvo como objetivo identificar la mortalidad por la enfermedad en Brasil en las primeras 6 semanas después de la confirmación del primer caso de muerte y delinear el perfil de estos individuos. Se trata de un estudio retrospectivo, descriptivo, transversal, que utilizó datos de los boletines diarios del Ministerio de Salud, analizando todos los casos de muertes ocurridas en Brasil por Covid-19, del 17 de marzo al 26 de abril de 2020. Se incluyeron datos de todos los estados de la federación y se caracterizaron por sexo, edad, color de piel y presencia de comorbilidad asociada. Entre las muertes, el 72\% fueron de personas mayores de 60 años, aunque alrededor del $80 \%$ de los infectados no pertenecían a este grupo de edad. Aún así, el 60\% eran hombres, incluso con un promedio de solo el 51,4\% de hombres infectados. La frecuencia de muertes fue significativamente mayor entre las personas blancas en comparación con las personas morenas y negras ( $\mathrm{p}<0,0001)$. La tasa de mortalidad fue del 6,92\%, siendo mayor entre los hombres, los ancianos - especialmente entre los que tenían morbilidades asociadas - y los de piel blanca.
\end{abstract}

Palabras clave: Covid-19; SARS-CoV-2; Mortalidad; Factores de riesgo.

\title{
1. Introdução
}

Em dezembro de 2019, a cidade de Wuhan, capital de Hubei, uma província na China, tornou-se o centro de um surto de pneumonia de causa desconhecida. Em 7 de janeiro de 2020, um cientista chinês isolara um novo coronavírus, causador de uma síndrome respiratória grave, ao qual ele chamou de coronavírus da síndrome respiratória aguda grave 2 (SARS-CoV-2) (Moreira, 2020; Teixeira et al., 2020), cuja enfermidade causada já era conhecida como 2019-nCoV e, mais tarde, em fevereiro de 2020, passou a doença pelo coronavírus 2019 (Covid-19) (Caramelo, 2020; OPAS, 2020; WHO, 2019).

Do dia 31 de dezembro de 2019, data em que a China comunicou a Organização Mundial da Saúde (OMS) sobre o surto da Covid-19, bastaram 93 dias para o mundo atingir 1 milhão de casos confirmados da doença, no 2 de abril. O segundo milhão de casos foi registrado 13 dias depois, em 15 de abril, e o terceiro milhão 12 dias depois, em 27 de abril (OPAS, 2020; WHO, 2019).

A Covid-19 já foi constatada em 185 países no planeta, com distribuição desigual pelos hemisférios. Apesar da subnotificação, os dados disponíveis apontam que países situados no Hemisfério Norte representam hoje $95 \%$ dos casos confirmados e $97 \%$ do total de 206.640 mortes. No Hemisfério Sul, onde se encontra a maior parte dos pais subdesenvolvidos, registram-se $5 \%$ dos casos confirmados e 3\% dos óbitos. Esses países abrigam $12 \%$ da população do mundo e, até 27 de abril de 2020, já registram cerca de 151 mil casos e 7.000 mortes por Covid-19 (WHO, 2019).

Em 26 de fevereiro de 2020, o Ministério da Saúde confirmou o primeiro caso no Brasil e, em meados de março, a primeira morte por coronavírus, ambos em São Paulo, embora o Ministério da Saúde tenha declarado que o coronavírus já circulava no Brasil desde janeiro de 2020. O número oficial de mortes provocadas pela Covid-19 no Brasil na manhã do dia 27 de abril era 4.298 e 63.328 casos da doença tinham sido confirmados em todo o país (Brasil, 2020). Porém, quem são essas vítimas? O que se sabe sobre elas? Alguns estudos têm mostrado que a mortalidade aumenta em indivíduos mais idosos e com doenças preexistentes (Zhou et al., 2020; Imanpour et al., 2020; Sullivan et al., 2015).

Foram identificados possíveis fatores de risco, como o alto escore Sequential Organ Failure Assessment (SOFA) e o dímero D superior a $1 \mu \mathrm{g} / \mathrm{mL}$ (Brasil, 2019). Entretanto, esses estudos foram realizados em outras populações, como asiáticas e europeias. É possível que uma análise detalhada do perfil do paciente que vai a óbito no Brasil possa contribuir para a tomada de decisões assertivas no tratamento dos pacientes infectados, principalmente porque a reação do organismo diante da infecção pelo novo coronavírus é diferente em cada indivíduo, podendo também o ser de população para população (Schmidt et al., 2009).

O objetivo deste estudo foi identificar a mortalidade por Covid-19 no Brasil nas 6 primeiras semanas após a confirmação do primeiro caso de óbito e traçar o perfil desses indivíduos. 


\section{Metodologia}

Trata-se de um estudo retrospectivo de abordagem quantitativa descritiva que utilizou dados dos boletins diários do Ministério da Saúde divulgados em seu site (Brasil, 2020). A população de estudo foi formada por todos os casos de óbitos ocorrido no Brasil por Covid-19 até a data de 26 de abril de 2020. Os dados foram buscados diariamente desde o dia 17 de março nos registros de óbito por Covid-19 no Brasil. Foram excluídos da análise os casos ainda sob investigação da causa do óbito e aqueles que, mesmo com sintomas típicos, não foram investigados.

Foram incluídos dados de todos os estados da federação e caracterizados por sexo, idade, cor da pele e presença de comorbidade associada. Também não houve distinção se o óbito ocorreu em ambiente hospitalar ou em casa.

Consideraram-se os óbitos das 6 primeiras semanas a partir 17 de março nos óbitos no por Covid-19 no Brasil. Isso porque, houve pico da pandemia, em diversas regiões, nas 2 semanas seguintes, ou seja, na sétima e oitava semana após a confirmação do primeiro óbito (Brasil, 2020). As comparações das frequências por características foram analisadas por meio do teste do qui-quadrado. Considerou $\mathrm{p}<0,05$ como significância estatística. Por utilizar dados públicos, a pesquisa foi dispensada de avaliação pelo Comitê de Ética em Pesquisa, conforme a resolução 510/2016 do Conselho Nacional de Saúde (CNS) (Brasil, 2016).

\section{Resultados}

De acordo com os dados apurados, $72 \%$ das mortes foram de pessoas com mais de 60 anos, embora cerca de $80 \%$ dos infectados não pertenciam a essa faixa etária. Entre os óbitos, $60 \%$ se deram no sexo masculino, embora apenas $51,4 \%$ dos infectados eram do sexo masculino. A hospitalização do paciente com Covid-19 foi um dos sinais de gravidade da doença: cerca de 7,2\% dos pacientes hospitalizados foram a óbito. Ainda, para a maioria das faixas etárias, ocorreram mais hospitalizações para o sexo masculino. Outra característica importante foi que $70 \%$ apresentavam pelo menos um fator de risco, sendo a cardiopatia a mais comum entre elas, a qual foi registrada em $40 \%$ dos casos. A frequência de óbitos foi significantemente maior entre os indivíduos de cor branca em relação aos de cor parda e negra $(\mathrm{p}<0,0001)($ Tabela 1).

Tabela 1 - Perfil do indivíduo que foi a óbito por Covid-19 no Brasil até 26 de abril de 2020, n=4.285.

\begin{tabular}{lcc}
\hline Características & $\mathbf{n}(\boldsymbol{\%})$ & Valor de p \\
\hline Idade, anos & $3.085(72)$ & $<0,0001$ \\
$\quad<60$ & $1.200(28)$ & \\
$>60$ & & $<0,0001$ \\
Sexo & $2.571(60)$ & \\
$\quad$ Homens & $1.714(40)$ & \\
Mulheres & & \\
Fator de risco & $2.999(70,2)$ & \\
Com & $1.286(29,8)$ & $<0,0001$ \\
Sem & & \\
Doenças cardíacas & $1.713(39,9)$ & \\
Com & $2.571(60,1)$ & $<0,0001$ \\
Sem & & $<0,0001$ \\
Raça & $2.400(56,2)$ & \\
Brancos & $1.457(34,1)$ & \\
Pardos & $215(5)$ & \\
Negros & & \\
\hline
\end{tabular}

Fonte: Brasil (2020). 
O primeiro caso de óbito notificado ocorreu em 12 de março de 2020. Entre os dias 20 e 30 de março, o número de óbitos aumentou 15 vezes. Nos quatro dias seguintes, o número dobrou de 165 para 328. O número dobrou novamente em 5 dias e, novamente, 7 dias depois, com manutenção desse período de tempo para dobrar o número de óbitos. O que mais chama atenção na figura 1 é o aumento muito grande do número de casos em pouco tempo, mostrado o rápido contágio da doença e sua grande capacidade de contágio (Figura 1).

Figura 1 - Frequência absoluta de óbitos por Covid-19 ao longo do tempo no Brasil.

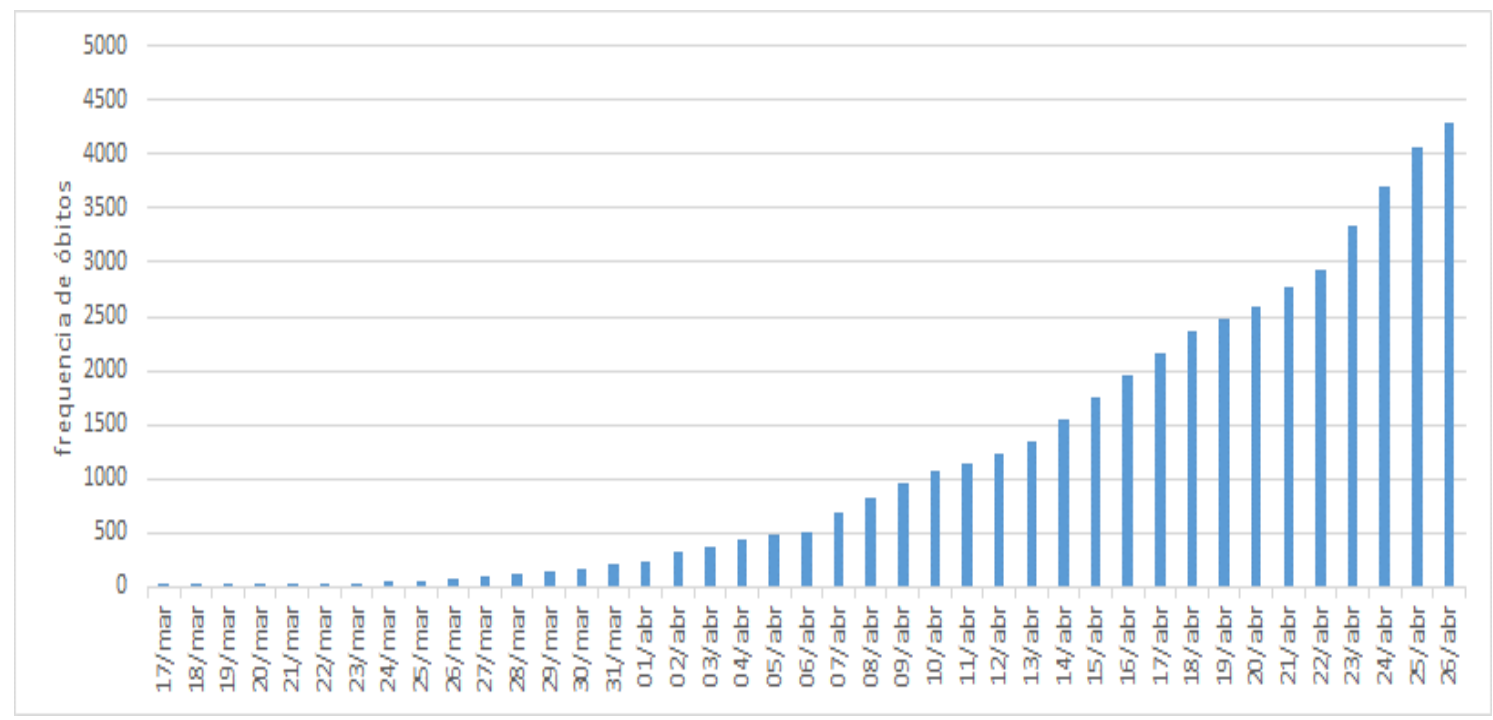

Fonte: Brasil (2020).

Foi possível extrais dos dados uma relação de óbitos a cada cem mil habitantes, observando-se que as quatro maiores relações foram ocupadas por cidade interioranas. As duas maiores cidades do país apresentaram a relação menor do que seis óbitos por cada cem mil habitantes. Portanto os óbitos ocorrem mais em lugares onde os serviços de saúde são mais deficitários, e aponta para as desigualdades nos equipamentos de saúde do país. (Figura 2).

Figura 2 - Ranque brasileiro de mortalidade por Covid-19por 100 mil.

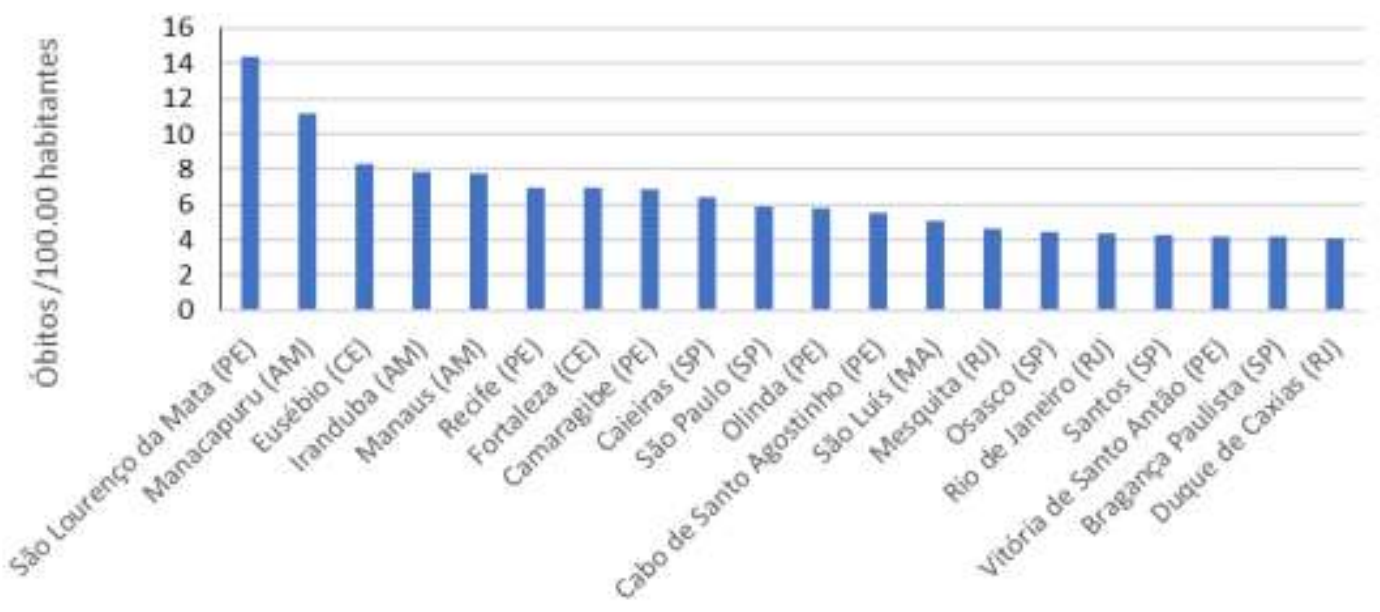

Fonte: Brasil (2020). 
O número de infectados relacionou-se com o de óbitos. Inicialmente, essa relação mostrou mortalidade de 0,34\%; em 10 dias, subiu para 2,72\%; nos 10 dias seguintes, aumentou para 5,03\%; em 17 de abril, a taxa de mortalidade já era de 6,44\% e taxa final, de 6,99\%. Estes dados apontam que a taxa de mortalidade aumentou porque houve superlotação dos leitos hospitalares já nesta data, além dos aumentos de casos não diagnosticados da doença. Ou seja, se aumenta os óbitos, mas aumenta também o número de casos não diagnosticados, a relação óbitos por infectados aumenta. (Figura 3).

Figura 3 - Relação número de infectados com o número de óbitos ao longo do tempo.

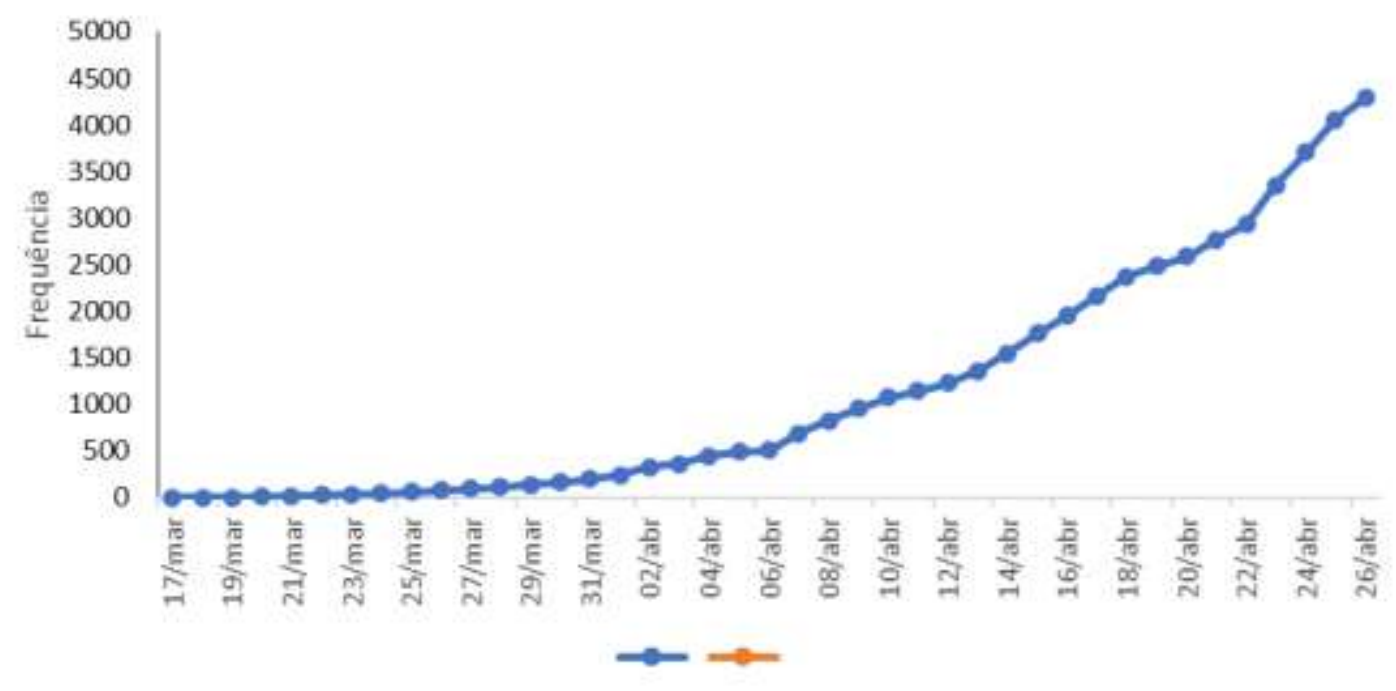

Fonte: Brasil (2020).

A hospitalização do paciente com Covid-19 foi um dos sinais de gravidade da doença. Cerca de 7,2\% dos pacientes hospitalizados foram a óbito. A Figura 4 mostra que, para a maioria das faixas etárias, ocorreram mais hospitalizações para o sexo masculino. Este fato constatado mais ainda necessita ser mais bem estudado para melhor compreensão.

Figura 4 - Frequência de hospitalização distribuída por sexo e idade.

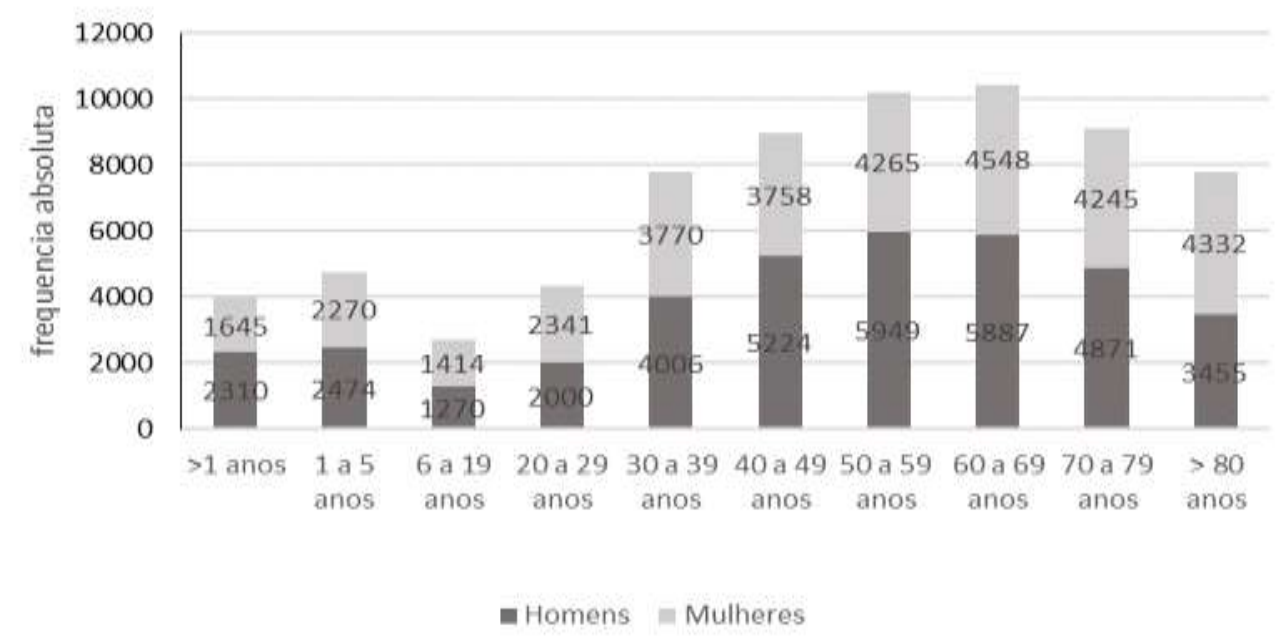

Fonte: Brasil (2020). 


\section{Discussão}

O objetivo deste estudo foi identificar a mortalidade por Covid-19 nas 6 primeiras semanas após a confirmação do primeiro caso da doença no Brasil e traçar o perfil desses indivíduos. Dentre os principais resultados, pode ser analisada, primeiramente, a taxa de mortalidade, que foi maior entre os indivíduos do sexo masculino, assim como entre os mais idosos, os que tinham morbidades associadas e aqueles de cor branca da pele. Posteriormente, verifica-se que o número de infectados dobrou a cada 5 dias no período estudado, e as taxas de mortalidade corrigida por 100 mil habitantes foram maiores em cidades interioranas. A taxa de infectados não foi diferente para os sexos.

A extração de dados divulgado por um órgão oficial tem sido um método para produção de pesquisas (Schimidt et al., 2009). Isso mostra que o método utilizado neste estudo é valido.

Desde que a pandemia se tornou uma realidade no mundo, sua taxa de letalidade tem atingido diferentes valores nos países. No Brasil, esse valor, até 26 de abril de 2020, era de 6,92\%, ou seja, bem superior aos 3,9\% da China, onde a doença começou. Porém, esse número está diretamente ligado à subnotificação dos casos confirmados no país. A expectativa é que, com o aumento de testes, a taxa de letalidade diminua. Quando se usam mais extensivamente os testes, encontram-se muitos indivíduos com quadros subclínicos positivos para o coronavírus. Isso aumenta o número de indivíduos com quadros leves, o que, por sua vez, diminui a letalidade (Teixeira et al., 2020). É possível que, com o passar dos dias e aumento dos números de testes feitos no Brasil, a taxa de mortalidade se reduza dentro de um padrão esperado.

No Brasil, a taxa mortalidade tem sido maior entre os homens do que entre as mulheres. O Centro de Controle e Prevenção de Doenças (CDC) da China realizou um estudo amplo utilizando-se os prontuários de 72.314 pacientes. Os dados mostraram que, dos casos confirmados, $51 \%$ eram homens, mas o que chamou a atenção dos especialistas é que a taxa de mortalidade também era maior para o sexo masculino: 2,8\% para os homens e 1,7\% para as mulheres (Caramelo et al., 2020). Outro estudo mostrou que o risco de mortalidade para homens é 2,15 vezes maior (Zhou et al., 2020).

$\mathrm{O}$ estrogênio pode ser a explicação para as diferentes respostas à doença. Ele pode estimular aspectos da imunidade importantes para eliminar uma infecção viral e responder bem às vacinas, contribuindo para maior imunidade do sexo feminino no surto atual de coronavírus, mas ainda não existem dados comprobatórios para essa afirmação (Imanpour et al., 2020). Além disso, as mulheres tendem a apresentar maior imunidade do que os homens após infecções virais, incluindo a gripe, o que deixaria a população feminina mais preparada para uma pandemia como a da Covid-19 (Sullivan et al., 2015). Embora a melhor resposta imune das mulheres contribua para eliminação mais rápida dos micróbios, essas respostas robustas também podem contribuir para uma maior suscetibilidade a doenças inflamatórias e autoimunes entre as mulheres.

Há ainda vários fatores sociais e culturais que podem aumentar a taxa de mortalidade nos homens. No Brasil, a taxa de tabagistas entre homens é maior do que entre as mulheres (Brasil, 2010). Como o coronavírus atinge principalmente os pulmões, causando pneumonia, o tabagismo aumenta o risco de complicações quando há uma infecção pelo coronavírus. Outro fator que tem impacto direto na taxa de mortalidade dos homens infectados pelo novo coronavírus é a existência de doenças prévias subdiagnosticadas, como diabetes e hipertensão em homens, ou mesmos as cardiopatias, que são, de fatos, mais prevalentes em homens (Schimidt et al., 2009). A causa mais provável teria a ver, então, com o estilo de vida. Ao redor do mundo, homens tendem a beber e a fumar mais do que as mulheres e, portanto, ficam mais suscetíveis a desenvolver doenças pulmonares e cardiopatias, o que os fragilizaria, caso contraíssem o coronavírus (Brasil, 2010; Schimidt et al., 2009).

As taxas de mortalidade aumentam gradualmente, de acordo com a idade: para pessoas com 40 anos, a taxa no Brasil é de $0,4 \%$; para a faixa etária dos 50 anos, $1,3 \%$; para a faixa etária dos 60 anos, 3,6\%; após os 70 anos, a taxa sobe para 8\%, e, na nona década de vida, ou seja, após 80 ou mais, sobe para 14,8\%. Dados da China indicaram que adultos mais velhos, particularmente os com sérias condições de saúde subjacentes, têm maior risco morte por Covid-19 do que os jovens (Teixeira 
et al., 2020). Embora a maioria dos casos relatados de Covid-19 na China tenha sido leve (81\%), aproximadamente $80 \%$ das mortes ocorreram entre adultos com idade $\geq 60$ anos; apenas uma $(0,1 \%)$ morte ocorreu em uma pessoa com idade $\leq 19$ anos (Zheng et al., 2020). Isso ocorre devido a condições subjacentes, como hipertensão, diabetes, doenças cardiovasculares e respiratórias crônicas e câncer (CDC, 2020).

Os dados deste estudo mostraram maior mortalidade entre os indivíduos de cor branca. O Ministério da Saúde inicialmente divulgou os dados por sexo e idade, mas passou a divulgar por raça/cor da pele. Cerca de $64 \%$ das mortes e $74 \%$ das hospitalizações por Covid-19 são de indivíduos de cor branca. Aparentemente, porém, a letalidade é maior entre os pardos e negros, já que eles representam um quarto de todas as hospitalizações e um terço de todos os óbitos (Zhou et al., 2020). Não há hipótese ainda sobre tais achados. Existem apenas alguns questionamentos, e, talvez, as subnotificações ou o número de casos ainda em investigação possam sanear estas dúvidas.

Outro dado importante encontrado por este estudo foi que o número de infectado dobra a cada 5 dias. Na Espanha e na Itália, epicentros da epidemia no mundo nos meses de março e abril, o número de casos dobrava a cada 6 dias (WHO, 2020). Isso aponta para a ineficiência do isolamento social adotado nos diversos estados brasileiros. É possível que a primeira e a segunda semanas de maio, as quais correspondem à sétima e à oitava semana após a confirmação do primeiro óbito no Brasil, os números de infectados e de óbitos cresçam semelhantemente aos da Itália e da Espanha.

Por fim, o número de óbitos corrigido por cem mil habitantes é maior em cidades interioranas. Tal fato também ocorreu em outros lugares do mundo (Remuzzi \& Remuzzi, 2020) e pode estar relacionado à falta de manejo e do tratamento adequando para pacientes com Covid-19 em cidades pequenas, que, em geral, contam com número reduzido de equipamentos de saúde ou indisponibilidade para seu uso efetivo para pacientes com Covid-19.

As limitações deste estudo estão ligadas ao fato de trabalharmos com dados disponibilizados pelos boletins diários do Ministério da Saúde, o que limita uma análise mais profunda tanto da mortalidade quanto do perfil dos indivíduos que foram a óbito. Entretanto, este estudo tem aplicabilidade clínica importante, a qual está principalmente relacionada ao fato de que homens, mais idosos, com comorbidades associadas e moradores de cidade pequenas e interioranas apresentam maior risco de mortalidade por Covid-19. Entretanto fica em aberto uma agenda de pesquisa em relação à mortalidade por raça/cor da pele.

\section{Conclusão}

Em análise detalhada a partir dos dados de mortalidade por Covid-19 no Brasil apresentados pelo Ministério da Saúde até o dia 26 de abril de 2020, observou-se que a taxa de mortalidade foi maior entre os indivíduos do sexo masculino, os mais idosos - principalmente entre aqueles que tinha morbidades associadas - e de cor branca da pele. Também o número de infectados dobrou a cada 5 dias no período estudado, e a taxa de mortalidade corrigida por 100 mil habitantes foi maior em cidades pequenas interioranas.

\section{Referências}

Caramelo, F., Ferreira, N., \& Oliveiros, B. (2020). Estimation of risk factors for COVID-19 mortality-preliminary results. MedRxiv. https://doi.org/10.1101/2020.02.24.20027268

Centers for Disease Control and Prevention (CDC). (2020). Severe Outcomes Among Patients with Coronavirus Disease 2019 (COVID-19)-United States, 2020. Morbidity and Mortality Weekly Report (MMWR), 69(12), 343-346. https://www.cdc.gov/mmwr/volumes/69/wr/mm6912e2.htm

Imanpour, H., Rezaee, H., \& Nouri-Vaskeh, M. (2020). Angiotensin 1-7: A novel strategy in COVID-19 Treatment. Advanced Pharmaceutical Bulletin, 10(4), $488-489$.

Ministério da Saúde. (2016). Resolução no 510/2016: Diário Oficial da União. https://bvsms.saude.gov.br/bvs/saudelegis/cns/2016/res0510_07_04_2016.html

Ministério da Saúde. (2019). Dados e números da prevalência do tabagismo, 2019: Ministério da Saúde. https://www.inca.gov.br/observatorio-da-politicanacional-de-controle-do-tabaco/dados-e-numeros-prevalencia-tabagismo 
Research, Society and Development, v. 10, n. 1, e34210111588, 2021 (CC BY 4.0) | ISSN 2525-3409 | DOI: http://dx.doi.org/10.33448/rsd-v10i1.11588

Ministério da Saúde. (2020). COVID-19. Painel coronavírus: Ministério da Saúde. https://covid.saude.gov.br/

Moreira, R. S. (2020). COVID-19: unidades de terapia intensiva, ventiladores mecânicos e perfis de mortalidade latente associados à letalidade no Brasil. Cadernos de Saúde Pública, 36 (5), e00080020.

Remuzzi, A., \& Remuzzi, G. (2020). COVID-19 and Italy: what next? The Lancet, 395 (10231), 1225-1228.

Organização Pan-Americana da Saúde (OPAS). (2020). Folha informativa COVID-19 -Escritório da OPAS e da OMS no Brasil. Brasília, DF: OPAS. https://www.paho.org/bra/index.php?option=com_content\&view=article\&id=6101:covid19\&Itemid=875

Schmidt, M. I., Duncan, B. B., Hoffmann, J. F., Moura, L. D., Malta, D. C., \& Carvalho, R. M. S. V. D. (2009). Prevalência de diabetes e hipertensão no Brasil baseada em inquérito de morbidade auto-referida, Brasil, 2006. Revista de Saúde Pública, 43, 74-82.

Sullivan, J. C., Rodriguez-Miguelez, P., Zimmerman, M. A., \& Harris, R. A. (2015). Differences in angiotensin (1-7) between men and women. American Journal of Physiology Heart and Circulatory Physiology, 308(9), H1171-6.

Teixeira, C. M., Madruga, G. D. A. M., Medeiros, G. B. D. S., Leite, J. G. T. M., \& Duarte, S. S. M. (2020). Análise comparativa das pandemias COVID-19 e H1N1. Brazilian Journal of Health Review, 3(2), 3591-3595.

World Health Organization (WHO). (2019). Coronavirus disease (COVID-19) pandemic. Geneve: WHO. https://www.who.int/emergencies/diseases/novelcoronavirus-2019

World Health Organization (WHO). (2020). Coronavirus disease 2019 (COVID-19) situation report. Geneva, Switzerland: WHO. https://www.who.int/emergencies/diseases/novel-coronavirus-2019/situation-reports

Zheng, Y. Y., Ma, Y. T., Zhang, J. Y., \& Xie, X. (2020). COVID-19 and the cardiovascular system. Nature Reviews Cardiology, 17(5), 259-260.

Zhou, F., Yu, T., Du, R., Fan, G., Liu, Y., Liu, Z., Xiang, J., Wang, Y., Song, B., Gu, X., Guan, L., Wei, Y., Li, H., Wu, X., Xu, J., Tu, S., Zhang, Y., Chen, H., \& Cao, B. (2020). Clinical course and risk factors for mortality of adult inpatients with COVID-19 in Wuhan, China: a retrospective cohort study. The Lancet, 395(10229), 1054-1062. 